\title{
Analysis of Active Learning Suitability of Subjects in Information and Electronics
}

\author{
https://doi.org/10.3991/ijep.v7i3.6968 \\ Yoshikatsu Kubota, Akiko Takahashi $\left.{ }^{\varpi}\right)$, Yoshihiro Hayakawa, \\ Yasuhiro Kashiwaba and Kuniaki Yajima \\ National Institute of Technology, Sendai College, Japan \\ yajima@sendai-nct.ac.jp
}

\begin{abstract}
Active Learning (AL)," the teaching method which puts emphasis on students' active participation in class and their abilities to discover problems and solve them, has been coming under the spotlight worldwide. NIT, Sendai College, Hirose Campus is promoting AL in the field of information and electronics. Especially, we are practicing " $\mathrm{A}^{3}$ Learning System" specializing in utilizing computers. We have aggressively introduced AL in class and seen the good effects of them. However, some problems are emerging in a certain type of subjects, which may mean that there are subjects unsuitable for AL. In this paper we report large-scale analysis of introduction of AL in information and electronics field and suggest that successful introduction of AL in class depends on the type of subjects.
\end{abstract}

Keywords-Active Learning; analysis; suitability; computing education

\section{Introduction}

Japan's educational system is undergoing radical change today. Japanese students are known to be passive learners. Actually, they are not so passive when they are young. In kindergarten and elementary school, children learn actively, think independently and enjoy working in pairs and groups. As they advance in grades in junior high school and high school, however, they gradually change their learning styles. Students quietly listen while teachers talk most of the time in class, and they copy in their notebook what teachers write on the blackboard. In high-ranked high schools, especially, which focus on preparing students for university entrance examinations, students' abilities are evaluated mainly in written tests to measure their knowledge, and teachers put an emphasis on teaching students as much knowledge as possible, which students try to memorize. Their top priority is to pass university entrance examinations, and those skills needed in the real world such as problem finding, problem solving, or communication skills are not fully developed in Japanese secondary education.

In recent years, "Active Learning (AL)," a teaching method which puts more emphasis on students' active participation in class and their abilities to discover and solve problems, has been coming under the spotlight worldwide [1] [2] [3] [4] [5] [6]. 
Ministry of Education, Culture, Sports, Science and Technology, Japan (MEXT) is also promoting the introduction of AL into Japanese schools and colleges to cultivate human resources who can think and act on their own [7]. Many high schools and universities all over Japan are now working on implementing AL.

NIT, Sendai College, Hirose Campus is promoting AL in the field of information and electronics. Especially, we are practicing "A3 Learning System" specializing in utilizing computers [8] [9]. The A3 Learning System comprises "Active Learning (AL)," "Problem/project Based Learning (PBL)" [10] [11] [12] and "Mastery Learning (ML)"[13] [14] [15] [16] [17], and uses ICT devices such as computers and tablet terminals. The A3 Learning System tries to boost students' motivation and personal growth. Students find problems, discuss how to solve them among themselves and develop some methods to achieve that goal in cooperation. Teachers support them by improving teaching systems and learning environments. AL includes activities in which students "learn by themselves or with their fellow students." A large amount of group work (pair work) and discussion is included. By utilizing ICT devices and electronic materials actively, we foster students' computer skills in regular classes. PBL cultivates students' thinking faculty, skills to solve problems, or creativity, and also their practical skills such as actual manufacturing and problem resolution using knowledge and technology as well as basic communication skills as a member of a team, which are necessary to carry out any project. By setting themes in a wide variety of fields, knowledge from each field is merged and deepened in computer field. ML is a style of individual learning in which each unit is learned at one's own pace. Once a unit is fully mastered, there is a transition to the next unit. Lessons consist of individual guidance and face-to-face lessons for support. (AL, PBL, and ML will all be referred to as AL in this paper.)

In each subject of information and electronics field, we have aggressively introduced AL in class and have seen their good effects. However, some problems are emerging in a certain type of subjects, which may mean that some subjects are not suitable for AL. In this paper we report large-scale analysis of introduction of AL in information and electronics field for computing education and suggest that successful introduction of $\mathrm{AL}$ in class depends on the type of subjects.

\section{Classification of Subjects in Information and Electronics Field}

\subsection{Overall Picture}

National Institute of Technology, Sendai College (NIT, Sendai College), Hirose Campus has three departments: Department of Intelligent and Electronic Systems, Department of Information Systems, and Department of Information Networks. The curriculum of each department is as follows.

In Department of Intelligent and Electronic Systems, students learn to understand how "intelligent" devices operate, and study widely element technology necessary for their development. In Department of Information Systems, students learn basics to 
application of information system step by step. In Department of Information Networks, students learn basics to application of information system and network system, mainly network technology and telecommunication technology in terms of conveyance and distribution of information.

According to the policy of these curricula, we are going to cultivate human resources who can think and act on their own by practice of A3 Learning System, as shown in Figure. 1.

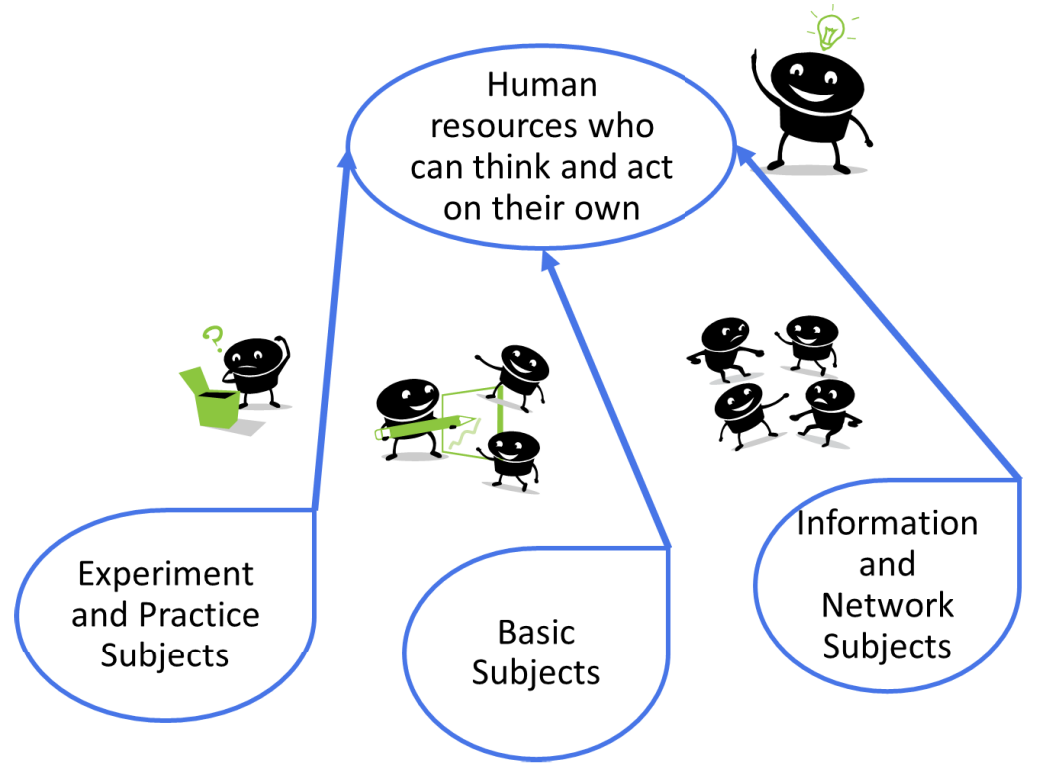

Fig. 1. The policy of these curricula

We have classified major subjects into those related to hardware technology, those related to software technology, and those related to network technology. However, the utilization of the Internet is essential today, considering how and when to use software and especially useful applications. Therefore, software technology and network technology are already closely connected with each other. Distribution of information on the Internet makes it possible to make a new classification of "information system," which fits with the times.

On the other hand, a curriculum focusing on electronics and electronic circuit will be needed in terms of network technology closely related to hardware, for example, high-frequency circuit, integrated circuit technology, or power-saving technology of devices. Moreover, sure and accumulated basic knowledge is essential in subjects of electric circuit, electronics circuit or electromagnetics to meet actual problems and the main part of curriculum needs to include basic contents. We can classify these as basic subjects essential in information and electronics field.

Moreover, in Department of Intelligent and Electronic Systems, one of the goals is to be able to control robots intelligently, and its curriculum starts with digital circuit technology and proceeds to microcomputer technology (including programming), 
embedded system, and finishes up with group-type PBL. In our campus, this type of curriculum means a group of mixed subjects of classroom lectures and experiments/practices, in other words, "spiral-type subjects", which is the strength of Japanese Kosens.

In this paper, the classification of subjects is especially important and we proceed with the classification into three fields, that is, basic subjects, experiment/practice subjects and information and network subjects (as it is suitable for the situation in our campus and also for computer education) as stated above. Actually, this classification is very good in terms of AL, as explained later in this paper. In the following sections, we explain subjects in each field.

\subsection{Basic Subjects}

In the lower grades in our college (16 to 18 years), Introduction to Electric Circuits, Electric Circuits, Fundamentals of Electronic Circuits, Introduction to Electromagnetics are offered as basics, and in the upper grades (when students are 19 and 20 years old), students study Electromagnetics A, Electromagnetics B, Electronic Circuits A, Electronic Circuits B, Circuit Engineering, Applied Physics I, Applied Physics II, Foundations of LSI Circuits, Advanced Electronic Circuits, Electronic Devices I, Electronic Devices II, Introduction to Electronic Properties of Materials, Electromagnetic Wave Engineering I, Electromagnetic Wave Engineering II, Electromagnetic Wave Engineering III.

In subjects on electric circuits, it is necessary to learn and use freely distinctive mathematics in circuit subjects such as how to solve circuit equation and methods of display and calculation in AC circuits, and to master these subjects, students need repeated practice.

In subjects on electronic circuit, it is necessary for students to understand basics of electric circuits beforehand. Introduction to Electric Circuit and Electric Circuit are also important, because Electronic Circuits, which is a subject in higher grades, presupposes the understanding of basics of electronic circuit.

The subjects on electromagnetics are also incremental, that is, students need to proceed step by step, accumulating pieces of specialized knowledge. Therefore, the introductory curriculum in the lower grades is extremely important.

\subsection{Experiment and Practice Subjects}

In the lower grades, Introduction to Digital Technology, Digital Technology, Introduction to Microcomputers, Project Practice, Fundamental Experiments, Experiments are offered as basics and Digital Systems A, Digital Systems B, Computer Systems, Embedded Systems, Digital Control, Computer Architecture, Network Architectures, Experiments I, Experiments II are offered in the upper grades.

Subjects on digital circuits are also cumulative and require students to learn step by step. Among these subjects, Boolean algebra and logical operation are necessary at introduction but they are relatively easy to learn. The subjects in this category are easy to work on as AL, since there are enough facilities for experiments and practices 
in our college and it is easy to put lectures and practices alternately and effectively in class.

Experiments offered in all the departments are all student-centered and they are AL-type subjects.

\subsection{Information and Network Subjects}

In the lower grades, Computer Literacy, Introduction to Programming, Programming, Advanced Programming I, Data Engineering, Foundations of Network Systems, Introduction to Networking are offered as basics, and in the upper grades, students study advanced Programming II, Applied Programming I, Applied Programming II, Fundamentals of Software Engineering, Network Programming I, Network Programming II, Networking I, Networking II, Networking III, Networking IV, Networking Technology I, Networking Technology II, Data Management Technology, Software Analysis and Design, Information Security, Foundations of Knowledge Engineering, Operating Systems, Information Theory, Network Theory, Multimedia Information, Information Security, Information Sociology, Distributed Computing I, Distributed Computing II, Network System Development, Computer Systems. Subjects on programming are mainly experiments and practices and it is relatively easy to introduce Mastery Learning into these subjects. We might have some difficulty in setting levels to each subject on information including Computer Science, but students do not always need cumulative knowledge in the field. It is possible to build a closed curriculum among the subjects.

\section{$3 \quad$ Analysis of introduction of AL into class Field}

\subsection{Assessment Standard for Introduction of AL into Class}

We conducted a questionnaire survey of all teachers in our college to analyze how they introduce AL into class and examined the percentage of classes in which AL was carried out. We did the survey three times; in 2014, 2015 and the first semester in 2016.

Figure 2 shows the questionnaire form used in the first semester, 2016. "Subject," "Type (Lecture, Practicum, Experiment, Lab course," "Class/Dept.," and "Instructor" are set on the top, "Contents" on the left, and "Frequency" on the right in one page to make it easier for respondents to answer and also for us to tally. In 2014 and 2015, the first two years of the introduction of AL in our college, we asked "How to conduct a class," "Attempts in class," or "Preparation for introducing AL" and had respondents write answers in their own words. It was possible to do the same survey online, but we considered it to be easier to collect answers in paper.

In $\mathrm{AL}$, students acquire course contents and knowledge by actively learning on their own. Teachers have to prepare a system for urging students to learn independently. It is also essential for students to understand course contents and for teachers to devise a way to establish course contents in students. Furthermore, it is desira- 
ble for teachers to be highly skilled in class management. Therefore, the questionnaire consists of three parts.

\begin{tabular}{|c|c|c|c|c|l|}
\hline FY & 2016 & Subject & & Type & $\begin{array}{l}\text { Lecture, Practicum, } \\
\text { Experiment, Lab course }\end{array}$ \\
\hline Class/Dept. & & Instructor & & \\
\hline
\end{tabular}

Check the frequency box in this class. You can check more than one box.

\begin{tabular}{|c|c|c|c|c|c|c|}
\hline \multirow{2}{*}{\multicolumn{2}{|c|}{ Contents }} & \multicolumn{5}{|c|}{ Frequency } \\
\hline & & \multirow{2}{*}{$\begin{array}{l}\text { Always } \\
\text { (5) }\end{array}$} & $\begin{array}{l}\text { Very Often } \\
\text { (4) }\end{array}$ & \multirow{2}{*}{$\begin{array}{c}\text { Sometimes } \\
\text { (3) }\end{array}$} & \multirow{2}{*}{$\begin{array}{l}\text { Rarely } \\
\text { (2) }\end{array}$} & \multirow{2}{*}{$\begin{array}{c}\text { Almost } \\
\text { Never } \\
\text { (1) }\end{array}$} \\
\hline \multirow{11}{*}{ Class style } & Investigative Learning & & & & & \\
\hline & Problem-based Learning & & & & & \\
\hline & Project-based Learning & & & & & \\
\hline & Discovery Learning & & & & & \\
\hline & Experience Learning & & & & & \\
\hline & Cooperative Learning (Pair Work, Group Work) & & & & & \\
\hline & Debate & & & & & \\
\hline & Mutual Teaching and Learning & & & & & \\
\hline & Checking Answers with Partners & & & & & \\
\hline & Giving Questions with Each Other & & & & & \\
\hline & Flipped Learning & & & & & \\
\hline \multirow{7}{*}{ Tools } & Reflection Card & & & & & \\
\hline & Utilizing Slides & & & & & \\
\hline & Utilizing ICT Tools & & & & & \\
\hline & Utilizing a Document Camera & & & & & \\
\hline & Utilizing Multimedia (Videos, DVDs) & & & & & \\
\hline & Utilizing Tablets (for Students) & & & & & \\
\hline & Utilizing Learning Management System (LMS) & & & & & \\
\hline \multirow{9}{*}{ Instruction methods } & $\begin{array}{l}\text { Clarify Lesson Framework such as Learning Goals } \\
\text { or Key Words at the Start of Each Lesson }\end{array}$ & & & & & \\
\hline & Awareness of Voice Volume & & & & & \\
\hline & Awareness of Intonation & & & & & \\
\hline & Awareness of Eye Contact & & & & & \\
\hline & Using Questions Effectively (Open Questions) & & & & & \\
\hline & Creative Use of Blackboard or Slides & & & & & \\
\hline & Rubric & & & & & \\
\hline & Review Sheet & & & & & \\
\hline & Frequency of Using AL in Class & & & & & \\
\hline
\end{tabular}

Fig. 2. The questionnaire form conducted in the first semester, 2016

(i) How to conduct classes (Class style). We include four question items of "Investigative Learning," "Cooperative Learning (Pair Work, Group Work)," "Flipped Learning" and "Problem-based Learning" to see if teachers are trying to improve students' abilities to act and investigate on their own and to share what they have learned with others, two items of "Discovery Learning" and "Experience Learning" to see if teachers are attempting to maintain students' motivation and to give students opportunities to understand the learning contents through discovery or experiences, and five items of "Debate," "Mutual Teaching and Learning," "Checking Answers with Partners," "Giving Question with Each Other," and "Project-based Learning" to see if teachers are working to develop students' abilities to communicate and cooperate with others.

(ii) Effective Tools to have students understand and learn the contents of classes (Tools). We include a question items of "Reflection Card" to see if teachers are 
try to devise ways to deepen students' understanding of course goals and contents, and six items to see if teachers are figuring out ways to attract students' attention, to facilitate classes and to help students' understanding of the learning contents.

(iii) Important skills for teachers to communicate with students in class (Instruction methods). Four items of "Clarify Lesson Framework such as Learning Goals or Key Words at the Start of Each Lesson," "Awareness of Voice Volume," "Awareness of Intonation," and "Awareness of Eye Contact" are included to see if teachers are conscious of the skills necessary to manage lessons effectively, and also five items of "Using Questions Effectively (Open Questions," "Creative Use of Blackboard or Slides," "Rubric," "Review Sheet," and "Frequency of Using AL in Class" are included to see the overall rates of AL among all classes.

Different teachers have different understanding of AL. Therefore, we expected that through reading and answering the questions, teachers who understand AL well would reflect on their teaching style while teachers who do not understand AL enough would deepen their knowledge of AL. We also included basic keywords related to AL in each question to enhance teachers' awareness of AL.

In 2014 questionnaire, we included a question "Are you introducing AL in your classes?" We judged whether or not teachers were introducing AL in class only by looking at their answers to this question. In 2015 and 2016 first semester questionnaires, we set 27 questions related to three parts mentioned above and asked the respondents to answer in a five-point scale from "Always" to "Almost Never." We calculated the total points of each subject, regarding "Always" as 5 points and "Almost Never" as 1 point, and if the sum of all items is 30 or more, we consider that AL is "introduced in class." Therefore 2015 and 2016 standard of assessment is stricter than that of 2014. The number of subjects answered is respectively 82 in 2014, 99 in 2014 and 59 in 2016.

\subsection{Categorizing the Subjects for Analysis}

As we mentioned in 2.1, our campus has three departments, and each department has its own curriculum according to its specialized area. We made six subject groups out of the curriculum of each department and analyzed them. The groups are 1) Electrical and Electronic Circuit group, 2) Experiment and Practicum group, 3) Software group, 4) Hardware group, 5) Network group, and 6) Other group. Table 1 shows the grouping of subjects about which we received an answer from the teacher in charge. The subjects in 1) Electrical and Electronic Circuit group are basic subjects mentioned in 2.2, those in 2) Experiment and Practicum group and in 3) Hardware group are experiment and practice subjects in 2.3, and those in 4) Software group and 5) Network group are information and network subjects in 2.4 .

\subsection{Results}

Figures 3 and 4 show the executing rates of AL in all the subjects related to information and electronics. The rates were higher in 2015 and 2016 than in 2014, when AL was introduced into our college, though the assessments were stricter in 2015 and 2016 than in 2014. 
Table 1. Categorizing the Subjects

\begin{tabular}{|c|c|}
\hline Category & Subject \\
\hline $\begin{array}{l}\text { Electrical and Electronic } \\
\text { Circuit group }\end{array}$ & $\begin{array}{l}\text { Introduction to Electric Circuits, Electric Circuits, } \\
\text { Fundamentals of Electronic Circuits, Electronic Circuits A, } \\
\text { Electronic Circuits B, Electronic Circuits C, Advanced } \\
\text { Electronic Circuits, Circuit Engineering, Introduction to } \\
\text { Electromagnetics, Electromagnetics A, Electromagnetics } \\
\text { B, Electromagnetic Wave Engineering II, Electromagnetic } \\
\text { Wave Engineering III, Ultrahigh Frequency Engineering }\end{array}$ \\
\hline $\begin{array}{l}\text { Experiment and } \\
\text { Practicum group }\end{array}$ & $\begin{array}{l}\text { Project Practice, Fundamental Experiments on Intelligent } \\
\text { Electronics, Intelligent Electronics Practice, Intelligent } \\
\text { Electronics Manufacturing, Fundamental Experiments on } \\
\text { Information Systems, Information System Experiments I, } \\
\text { Information System Experiments II, Information System } \\
\text { Practice I, Information System Practice II, Fundamental } \\
\text { Experiments on Communication }\end{array}$ \\
\hline Software group & $\begin{array}{l}\text { Computer Literacy, Fundamentals of Computer } \\
\text { Systems, Foundations of Programming, Programming, } \\
\text { Applied Programming I, Applied Programming II, } \\
\text { Advanced Programming, Network Programming I, } \\
\text { Network Programming II, Distributed Computing I, } \\
\text { Distributed Computing II, Data Engineering, Fundamentals } \\
\text { of Data Engineering, Data Management Technology, } \\
\text { Information Theory, Information Engineering, } \\
\text { Fundamentals of Information Security, Information } \\
\text { Security, Software Analysis and Design, Fundamentals of } \\
\text { Software Engineering, Foundations of Knowledge } \\
\text { Engineering, Operating Systems, Numerical Calculation, } \\
\text { Information Mathematics, Multimedia Information }\end{array}$ \\
\hline Hardware group & $\begin{array}{l}\text { Introduction to Digital Technology, Digital Technology, } \\
\text { Introduction to Microcomputers, Microcomputers I, } \\
\text { Microcomputers II, Embedded Systems, Digital Systems A } \\
\text {, Digital Systems B, Computer Systems, Computer } \\
\text { Architecture }\end{array}$ \\
\hline Network group & $\begin{array}{l}\text { Introduction to Networking, Networking Technology I, } \\
\text { Networking Technology II, Networking I, Networking II, } \\
\text { Networking III, Networking IV, Network Theory, } \\
\text { Foundations of Network Systems, Network System } \\
\text { Development, Network Architectures }\end{array}$ \\
\hline Others & $\begin{array}{l}\text { Introduction to Electronic Devices and Systems, } \\
\text { Electronic Devices and Systems, Electronic Measurement, } \\
\text { Information Sociology, Optical Communication Systems, } \\
\text { Wireless Communication Systems, Laws of } \\
\text { Communication, Communication Systems, Welfare } \\
\text { Engineering }\end{array}$ \\
\hline
\end{tabular}




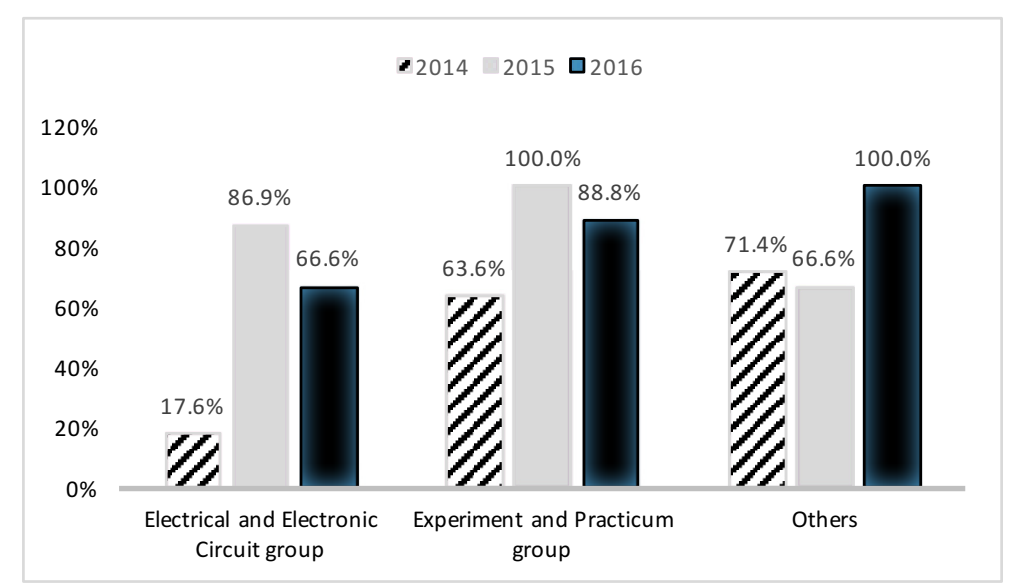

Fig. 3. Electrical and Electronic Circuit Group and Experiment and Practicum Group

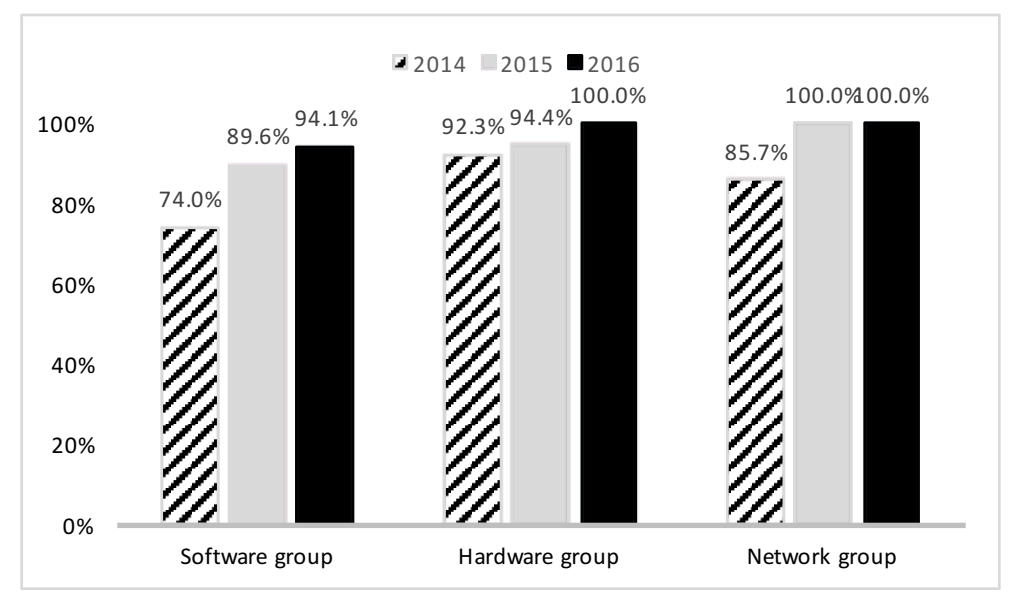

Fig. 4. Main groups

Electrical and Electronic Circuit Group had the lowest executing rate of 17.6 percent in 2014 but the rate sharply increased to 85.6 percent in 2015 . The executing rate of AL in Experiment and Practicum Group increased from 63.6 percent in 2014 to 100 percent in 2015 . The rates in both groups dropped to 66.6 percent and 88.8 percent respectively in 2016. The rate in the former group dropped because three subjects of this group were considered to be "not introducing AL in class" in 2016. These subjects scored just over 30 in 2014 and 2015. Only a few points dropped in these subjects in 2016 and they went below 30, so they were regarded as "not introducing AL in class." We think that the teachers' attempts to adjust and improve the way of conducting the class affected the results. The rate in the latter group dropped because one subject was regarded as "not introducing AL in class." This subject is similar to Graduation Research. The questionnaire was intended for ordinary subjects conducted in classroom, so this subject was not suitable for the questionnaire. Taking those into 
consideration, all subjects in this group can be regarded as "fully introducing AL in class."

On the other hand, Software Group, Hardware Group and Network Group showed steady improvement in the executing rates from 2014 to 2016 as Figure 4 shows.

\subsection{Discussion}

2015 and 2016, we considered a subject to be "introducing AL in class" when the sum of all question items was 30 or more. However, the subjects whose points were just below or above 30 could be judged as "introducing AL in class" or "not introducing AL in class" by a small fluctuation of a few points, so we take a look at the mean value of the sum of all question items (see Figure 5 and Figure 6). We need to take it into consideration that the data of 2016 is that of the first semester, not of the whole year.

The transition of the mean value shows that all the groups except for Network group improved in terms of introducing AL into class. As for Network group, the number of subjects in 2016 was limited, because they were the subjects conducted only in the first semester. The mean value of the same subjects in 2015 was 44.5 , so the mean value of this group slightly improved in 2016 compared with that in 2015 . To be more precise, we show the distributions of points in each group.

As Figure 3 shows, the subjects in Electrical and Electronic Circuit group were low in executing rates in 2014, and the teachers teaching these subjects may have thought it difficult to introduce AL into them. In 2015, the teachers seem to have made some attempts to introduce AL into more subjects of this group, but still the rates were 10 points or more lower than those of the other groups, as Figure 5 and 6 show. Figure 7 shows the change in the distribution of points in 2015 and in 2016. The distribution of points moved to the left in 2016, which may mean that these subjects are difficult to introduce AL into, although two subjects have extremely high points of 110 , which contributes to raising the mean of this group. We need to check these subjects more closely.

Figure 8 shows the distribution in Experiment and Practicum group. As we mentioned in 3.3, the subject with the lowest point of 5 is not an ordinary subject conducted in classroom, so we can say that the subjects in this group are "introducing AL in class." The subjects in this group require students to work on their own or work in groups, so the results were predictable.

Figure 9 shows the distribution of subjects in Software group. Most subjects in this group can also be regarded suitable for AL. The subjects with relatively low points are those related to programming, and those with relatively high points are information related subjects. Programming-related subjects require students to develop skills but may not need various methods of AL.

Figure 10 shows the distribution of points in Hardware group. Digital technology or embedded systems education are the typical subjects of "spiral-type education," which Japanese Kosens have aggressively promoted, so these subjects were AL in the first place or are easy to change into AL. 
Paper-Analysis of Active Learning Suitability of Subjects in Information and Electronics

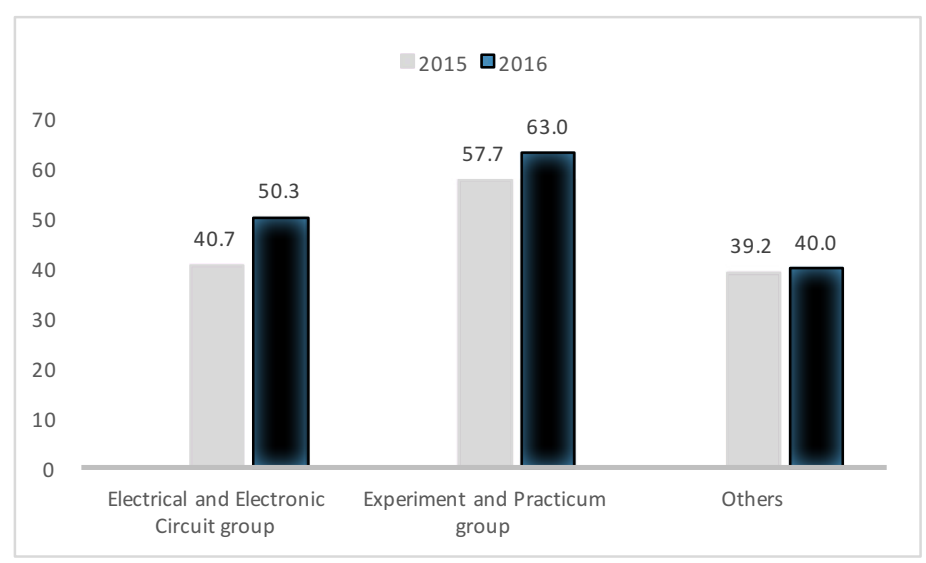

Fig. 5. Electrical and Electronic Circuit group and Experiment and Practicum group

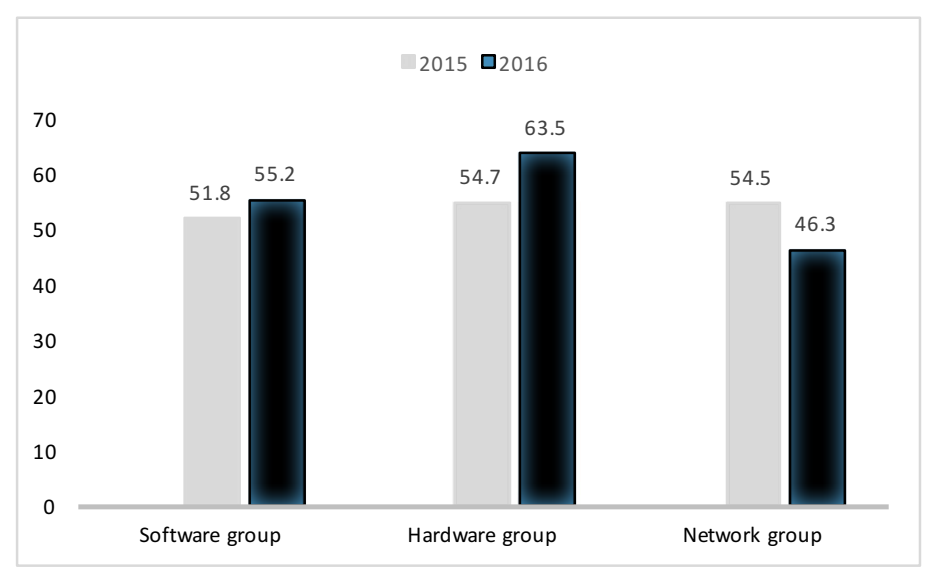

Fig. 6. Main groups

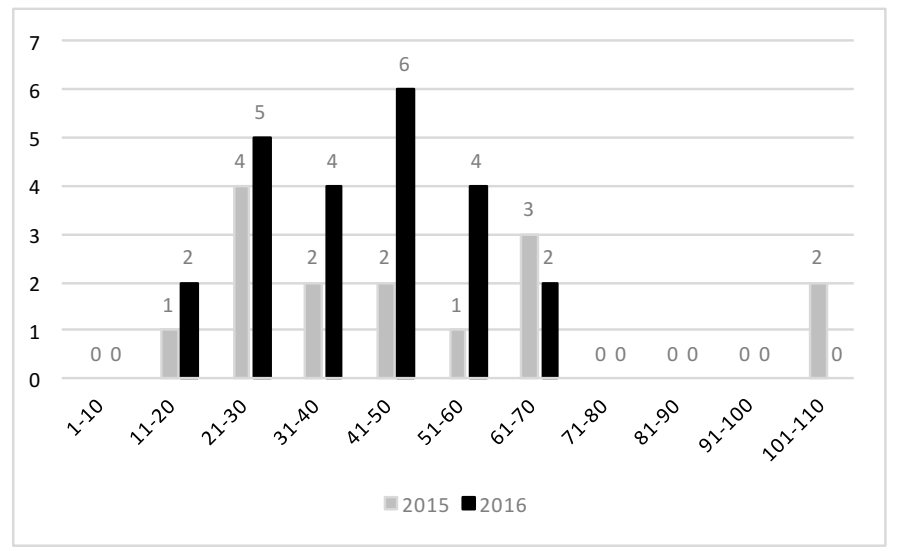

Fig. 7. Electrical and Electronic Circuit group 
Paper-Analysis of Active Learning Suitability of Subjects in Information and Electronics

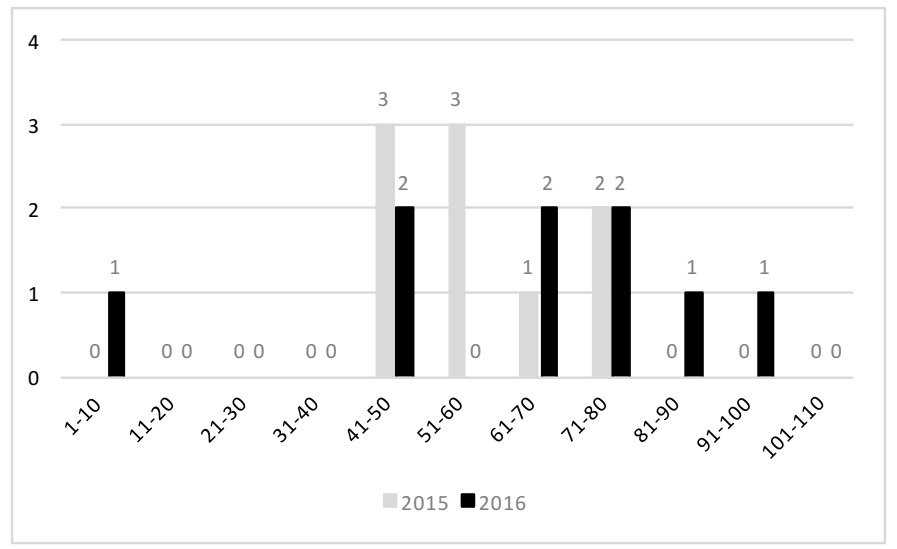

Fig. 8. Experiment and Practicum group

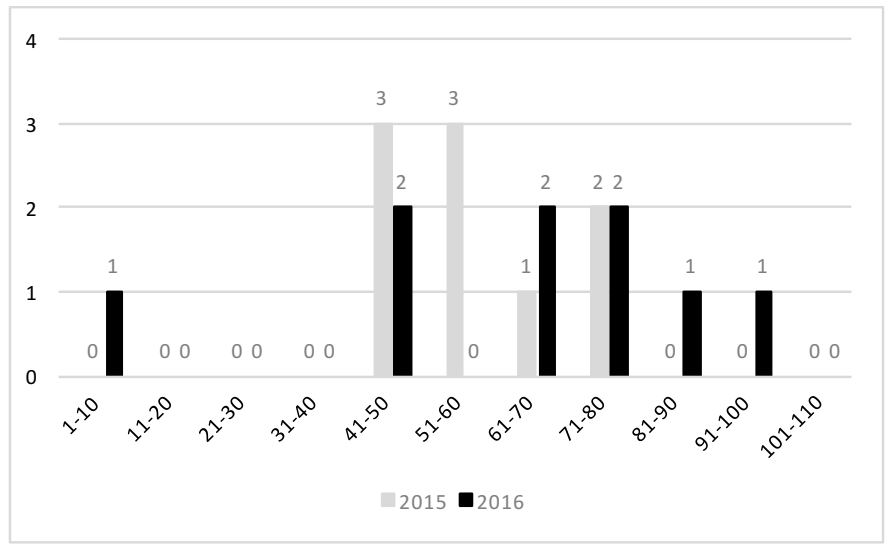

Fig. 9. Software group

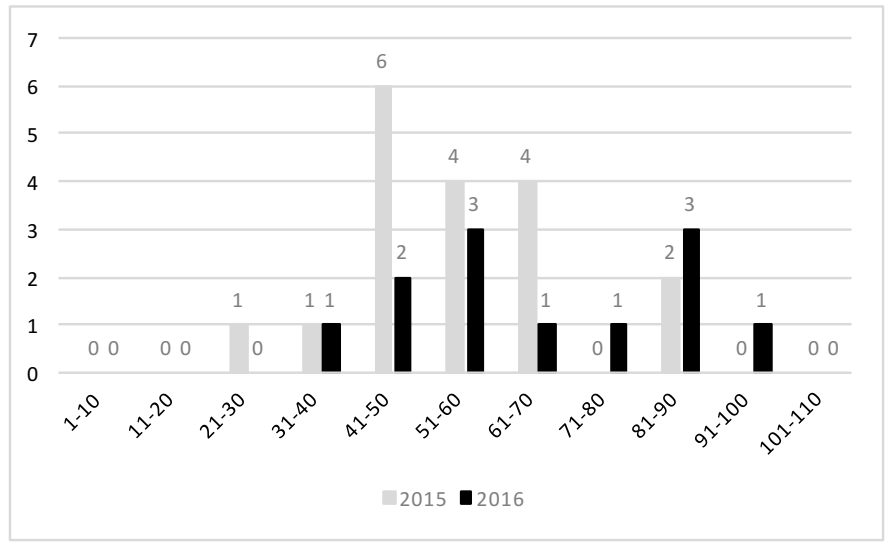

Fig. 10.Hardware group 
CompTIA CTT + , which is an international qualified certification of instructor kills, prepares knowledge and skills for instructors to realize good results in teaching such as "management of physical environment," "establishing and maintaining trust in learners," "improvement of motivation to master," "evaluation of achievement," or "conducting various teaching methods." They are good skills for AL teachers to learn. It does not exclude one-way lectures. Lectures are said to be effective in conveying a lot of knowledge within limited time. In Electrical and Electronic Circuit group (basic subjects), students cannot go on to learning actively unless they acquire a certain level of basic knowledge. We need to take this point into consideration and analyze the results further in detail.

On the other hand, Experiment and Practicum group, Software group, Hardware group, and Network group show the high executing rates of AL. In these subjects, socalled "spiral-type teaching method" had been conducted even before the introduction of AL into our curriculum. In this method, students learn some knowledge in lectures and then they understand it deeply by doing practice and experiments using the knowledge. In other words, it is a teaching method based on PDCA (Plan-Do-CheckAct) cycle. Therefore, these subjects can be suitable for AL.

We can see from the above analysis that some basic subjects are not suitable for AL but suitable for one-way lectures, because students need to acquire knowledge rather than learning actively and independently. However, some elements of ML can be applied to these subjects, for example, making sure that students learned necessary knowledge by testing over a certain period of time. By doing so, we consider that we can encourage students to learn actively.

\section{Conclusion}

In this paper we report large-scale analysis of introduction of AL into information and electronics field for computing education and suggest that successful introduction of AL into class depends on the type of subjects. The results of the questionnaire survey for the executing rates of $\mathrm{AL}$ in special subjects in information and electronics field shows that basic subjects are not suitable for AL because they require students to learn fundamental knowledge and it is easier for teachers to teach and for students to learn in a one-way method like lectures .

We would like to improve the contents of questionnaire to survey the executing rates of AL and establish methods of evaluating effectiveness of AL in a quantitative way as our next step.

\section{Acknowledgment}

This research was partially funded through the financial assistance of The Ministry of Education, Culture, Sports, Science and Technology Acceleration Program for University Education Rebuilding. 


\section{References}

[1] H. Niemi, 'Active learning - a cultural change needed in teacher education and schools,' Teaching and Teacher Education, vol. 18, pp. 763-780, 2002. https://doi.org/10.1016/S07 42-051X(02)00042-2

[2] M.W. Keyser, 'Active learning and cooperative learning: understanding the difference and using both styles effectively,' Research Strategies, Vol.17, pp.35-44, 2000. https://doi.org/10.1016/S0734-3310(00)00022-7

[3] N.F. Hassan, S. Puteh, and R. Buhari, 'Student understanding through the application of technology enabled Active Learning in practical training,' Procedia - Social and Behavioral Sciences, Vol. 204, pp. 318-325, 2015. https://doi.org/10.1016/j.sbspro.2015.08.158

[4] N.M. Mingazova, 'Modification of the Active Learning methods in environmental education in Russian universities,' Procedia - Social and Behavioral Sciences, Vol. 131, pp. 8589, 2014. https://doi.org/10.1016/j.sbspro.2014.04.083

[5] S. Freeman, S.L. Eddya, M. McDonougha, M.K. Smithb, N. Okoroafora, H. Jordta, and M.P. Wenderotha, 'Active learning increases student performance in science, engineering, and mathematics,' PNAS, Vol.111, no.23, pp.8410 - 8415, 2014. https://doi.org/10.1073/ pnas. 1319030111

[6] S.L. Eddy, M. Converse, and M.P. Wenderoth, 'PORTAAL: A Classroom Observation Tool Assessing Evidence-Based Teaching Practices for Active Learning in Large Science, Technology, Engineering, and Mathematics Classes,' CBE-Life Sciences Education, Vol. 14, pp.1-16, 2015. https://doi.org/10.1187/cbe.14-06-0095

[7] '2014 White Paper on Education, Culture, Sports, Science and Technology, ' Ministory of Education, Culture, Sports, Science and Technology-JAPAN, http://www.mext.go.jp/b_menu/hakusho/html/hpab201401/1376911.htm

[8] Akiko Takahashi, Yasuhiro Kashiwaba, Toshiaki Okumura, Toshihiko Ando, Kuniaki Yajima, Yoshihiro Hayakawa, Motomu Takeshige and Tatsuo Uchida, "Design of Advanced Active and Autonomous Learning System for Computing Education - A3 Learning System -," Proceedings of 2015 IEEE International Conference on TALE, pp.77-82, 2015. https://doi.org/10.1109/TALE.2015.7386020

[9] Akiko Takahashi, Yasuhiro Kashiwaba, Toshiaki Okumura, Toshihiko Ando, Kuniaki Yajima, Yoshihiro Hayakawa, Motomu Takeshige and Tatsuo Uchida, "A3 Learning System: Advanced Active and Autonomous Learning System," International Journal of Engineering Pedagogy (iJEP), submitted (12 Apr 2016, accepted). https://doi.org/10.3991/ ijep.v6i2.5645

[10] Jacobo Rodri'guez, Ana Lavero'n-Simavilla, Juan M. del Cura, Jose' M. Ezquerro, Victoria Lapuerta, and Marta Cordero-Gracia, 'Project Based Learning experiences in the space engineering education at Technical University of Madrid,' Advances in Space Research, Vol. 56, pp. 1319-1330, 2015. https://doi.org/10.1016/j.asr.2015.07.003

[11] L. Zhiyu, 'Study on the cultivation of college students' science and technology innovative ability in electrotechnics teaching based on PBL Mode,' IERI Procedia, Vol. 2, pp. 287292, 2012. https://doi.org/10.1016/j.ieri.2012.06.090

[12] Sandra Raquel Gonçalves Fernandes, 'Preparing graduates for professional practice: findings from a case study of Project-based Learning (PBL),' Procedia - Social and Behavioral Sciences, Vol. 139, pp. 219-226, 2014. https://doi.org/10.1016/j.sbspro.2014.08.064

[13] K. Kularbphettonga, P. Kedsiributa, and P. Roonrakwit, 'Developing an adaptive WebBased Intelligent Tutoring System using Mastery Learning technique,' Procedia - Social and Behavioral Sciences, Vol. 191, pp. 686-691, 2015. https://doi.org/10.1016/j.sbs pro.2015.04.619 
[14] M.E. Damavandi, and Z.S. Kashani, 'Effect of mastery learning method on performance and attitude of the weak students in chemistry,' Procedia Social and Behavioral Sciences, Vol. 5, pp. 1574-1579, 2010. https://doi.org/10.1016/j.sbspro.2010.07.327

[15] N. Shafie, T.N.T. Shahdan, and M.S. Liew, 'Mastery Learning Assessment Model (MLAM) in teaching and learning mathematics,' Procedia Social and Behavioral Sciences, Vol. 8, pp. 294-298, 2010. https://doi.org/10.1016/j.sbspro.2010.12.040

[16] G. Gladding, B. Gutmann, N. Schroeder, and T. Stelzer, 'Clinical study of student learning using mastery style versus immediate feedback online activities,' PHYSICAL REVIEW SPECIAL TOPICS - PHYSICS EDUCATION RESEARCH, Vol. 11, 010114, 2015. https://doi.org/10.1103/PhysRevSTPER.11.010114

[17] C.L.C. Kulik, J.A. Kulik, and R.L. Bangert-Drowns, 'Effectiveness of Mastery Learning Programs: A Meta-Analysis,' REVIEW OF EDUCA'TIONAL RESEARCH Summer, Vol. 60 no.2 265-299, 1990.

\section{Authors}

Yoshikatu Kubota is with Department of General Engineering at National Institute of Technology, Sendai College. His current research focuses on EFL (English as a Foreign Language), especially, vocabulary learning/teaching.

Akiko Takahashi is with Department of General Engineering at National Institute of Technology, Sendai College. Her current research focuses on multiagent system and mechanism design theory.

Yoshihiro Hayakawa is with Department of General Engineering at National Institute of Technology, Sendai College. His current research focuses on a huge size neural network to deal with real-world combinatorial optimization problems.

Yasuhiro Kashiwaba is with Department of General Engineering at National Institute of Technology, Sendai College. His current research focuses on electronic devices.

Kuniaki Yajima is with Department of General Engineering at National Institute of Technology, Sendai College. His current research focuses on web based educational system.

This article is a revised version of a paper presented at the 2016 IEEE International Conference on Teaching, Assessment and Learning for Engineering (TALE2016), held 7-9 December 2016, at Dusit Thani Bangkok Hotel, Bangkok, Thailand. Article submitted 31 March 2017. Published as resubmitted by the authors 12 May 2017. 